\title{
COCHRANE CORNER \\ Educational and skills-based interventions for preventing relationship and dating violence in adolescents and young adults
}

${ }^{\dagger}$ This review is an abridged version of a Cochrane review previously published in the Cochrane Database of Systematic Reviews, 2013, June 19, Issue 6: CD004534 (doi: 10.1002/ 14651858.CD004534.pub3) (see www. Cochranelibrary.com for information). Cochrane reviews are regularly updated as new evidence emerges and in response to feedback, and the Cochrane Database of Systematic Reviews should be consulted for the most recent version of the review.

(C) 2019 The Cochrane Collaboration. Published by John Wiley \& Sons Ltd.

We thank the Cochrane Review Group for their support in publishing these reviews.

See commentary in this issue.

\author{
Gracia L.T. Fellmeth, Catherine Heffernan, Joanna Nurse, Shakiba Habibula \& Dinesh Sethi
}

\section{Background}

Educational and skills-based interventions are often used to prevent relationship and dating violence among young people.

\section{Objectives}

To assess the efficacy of educational and skills-based interventions designed to prevent relationship and dating violence in adolescents and young adults.

\section{Search methods}

We searched the Cochrane Central Register of Controlled Trials (CENTRAL), MEDLINE, EMBASE, CINAHL, PsycINFO, six other databases and a trials register on 7 May 2012. We handsearched the reference lists of key articles and two journals (Journal of Interpersonal Violence and Child Abuse and Neglect). We also contacted researchers in the field.

\section{Selection criteria}

Randomised, cluster-randomised and quasi-randomised studies comparing an educational or skills-based intervention to prevent relationship or dating violence among adolescents and young adults with a control.

Data collection and analysis

Two review authors independently assessed study eligibility and risk of bias. For each study included in the meta-analysis, data were extracted independently by G.F. and one other review author (either C.H., J.N., S.H. or D.S.). We conducted meta-analyses for the following outcomes: episodes of relationship violence, behaviours, attitudes, knowledge and skills.

We included 38 studies (15 903 participants) in this review, 18 of which were cluster-randomised trials (11 995 participants) and two were quasi-randomised trials (399 participants). We included 33 studies in the meta-analyses. We included eight studies
(3405 participants) in the meta-analysis assessing episodes of relationship violence. There was substantial heterogeneity $\left(P^{2}=57 \%\right)$ for this outcome. The risk ratio was 0.77 (95\% confidence interval (CI) 0.53-1.13). We included 22 studies (5256 participants) in the meta-analysis assessing attitudes towards relationship violence. The standardised mean difference (SMD) was $0.06(95 \% \mathrm{Cl}-0.01$ to 0.15$)$. We included four studies (887 participants) in the meta-analysis assessing behaviour related to relationship violence; the SMD was -0.07 (95\% Cl -0.31 to 0.16 ). We included 10 studies (6206 participants) in the meta-analysis assessing knowledge related to relationship violence; the results showed an increase in knowledge in favour of the intervention (SMD $=0.44,95 \% \mathrm{Cl} 0.28-0.60$ ) but there was substantial heterogeneity $\left(P^{2}=52 \%\right)$. We included seven studies (1369 participants) in the meta-analysis assessing skills related to relationship violence. The SMD was $0.03(95 \% \mathrm{Cl}-0.11$ to $0.17)$. None of the included studies assessed physical health, psychosocial health or adverse outcomes. Subgroup analyses showed no statistically significant differences by intervention setting or type of participants. The quality of evidence for all outcomes included in our meta-analysis was moderate owing to an unclear risk of selection and detection bias and a high risk of performance bias in most studies.

\section{Authors' conclusions}

Studies included in this review showed no evidence of effectiveness of interventions on episodes of relationship violence or on attitudes, behaviours and skills related to relationship violence. We found a small increase in knowledge but there was evidence of substantial heterogeneity among studies. Further studies with longer-term follow-up are required, and study authors should use standardised and validated measurement instruments to maximise comparability of results. 